\title{
Effects of supercritical carbon dioxide extraction parameters on virgin coconut oil yield and medium-chain triglyceride content.
}

\begin{abstract}
The extraction of coconut oil has been performed using supercritical carbon dioxide (SC$\mathrm{CO} 2$ ). The extractions were performed at pressure and temperature ranges of 20.7-34.5 MPa and $40-80{ }^{\circ} \mathrm{C}$, respectively. It was observed that almost all (more than 99\%) of the total oil could be extracted. Response surface methodology (RSM) was applied to evaluate the effects of the parameters (pressure, temperature and $\mathrm{CO} 2$ consumption) on the extraction yield and medium-chain triglycerides (MCTs), in terms of the fatty acid content in the extracted oil. A correlation was established with p-values for both responses significant at the $95 \%$ confidence level.
\end{abstract}

Keyword: Virgin coconut oil; Medium chain triglycerides; Supercritical CO2; Extraction; Response surface methodology. 